\title{
NUMERICAL INVESTIGATIONS OF MIXED CONVECTION OF INCOMPRESSIBLE VISCOUS FLUID IN LNG STORAGE WITH A VARIOUS LOCATIONS OF INPUT AND OUTPUT MASS
}

\author{
Kristina A. Sklyarenko ${ }^{1}$, Ekaterina A. Lutceva ${ }^{1}$, Vyacheslav I. Maksimov ${ }^{1 a}$, Tatiana A. Nagornova ${ }^{1}$ \\ ${ }^{1}$ National Research Tomsk Polytechnic University, Tomsk 634050, Russia
}

\begin{abstract}
The article shows the results of mathematical simulation of mixed convection in the low-temperature storage of liquefied natural gas with a regenerative cooling. The regimes of mixed convection in a closed area with the different arrangement of the input and output sections of the masses are investigated. Two-dimensional nonstationary problem in the model of the Navier-Stokes in dimensionless variables "vorticity - stream function - temperature" was examined. Are obtained distributions of the hydrodynamic parameters and temperatures, characteristic basic laws governing the processes being investigated. Detailed circulating currents and carried out analysis of the mechanism of vortices formation and the temperature distribution in the solution for mixed convection mode with low Reynolds and Grashof numbers $\left(\mathrm{Gr}=10^{6}, 100<\mathrm{Re}<1000\right)$. Is established the significant influence of the geometrical arrangement of the input and output mass sections and input stream velocity on the structure of liquid flow and temperature in the low temperature LNG storage tanks.
\end{abstract}

\section{Introduction}

Study convection in the storage of liquefied natural gas (LNG) is of great importance in the simulation and optimization of production, storage and transportation of liquid substances [1-3].

Solving problems of convective heat transfer in the storage tanks of liquefied natural gas is necessary to predict the process of stratification of the liquid and the subsequent sudden stirring (the phenomenon of «rollover») to prevent technogenic emergency on such objects [4-6].

Is significant the analysis of the influence of the position of the sections of input and output on the flow pattern and with respect to heat- and mass exchange into the low-temperature storage tanks LPG. The objective is mathematical modeling of mixed convection of liquefied natural gas in a typical lowtemperature storage tank with local sources of input and output mass and taking into account the nonuniform heat transfer to the external borders of the region.

\section{Statement of the problem and the method of solution}

\footnotetext{
${ }^{\mathrm{a}}$ Corresponding author : elf@tpu.ru
} 
Is examined the flow of the incompressible viscous fluid and heat exchange into the cavity with two vertical, one horizontal of final thickness wall and one free of surface, with two sections of input and output of liquid (fig.1).

The thermophysical properties of medium do not depend on temperature, and flow regime is laminar. The fluid is considered Newtonian incompressible and satisfies the Boussinesq approximation.

The process of heat transfer in the test solutions is described by non-stationary Navier-Stokes equations [7-10]. To determine the flow and temperature fields in the environment used twodimensional non-stationary convection in the Boussinesq approximation in the variables «vorticity stream function - temperature» [7-10].

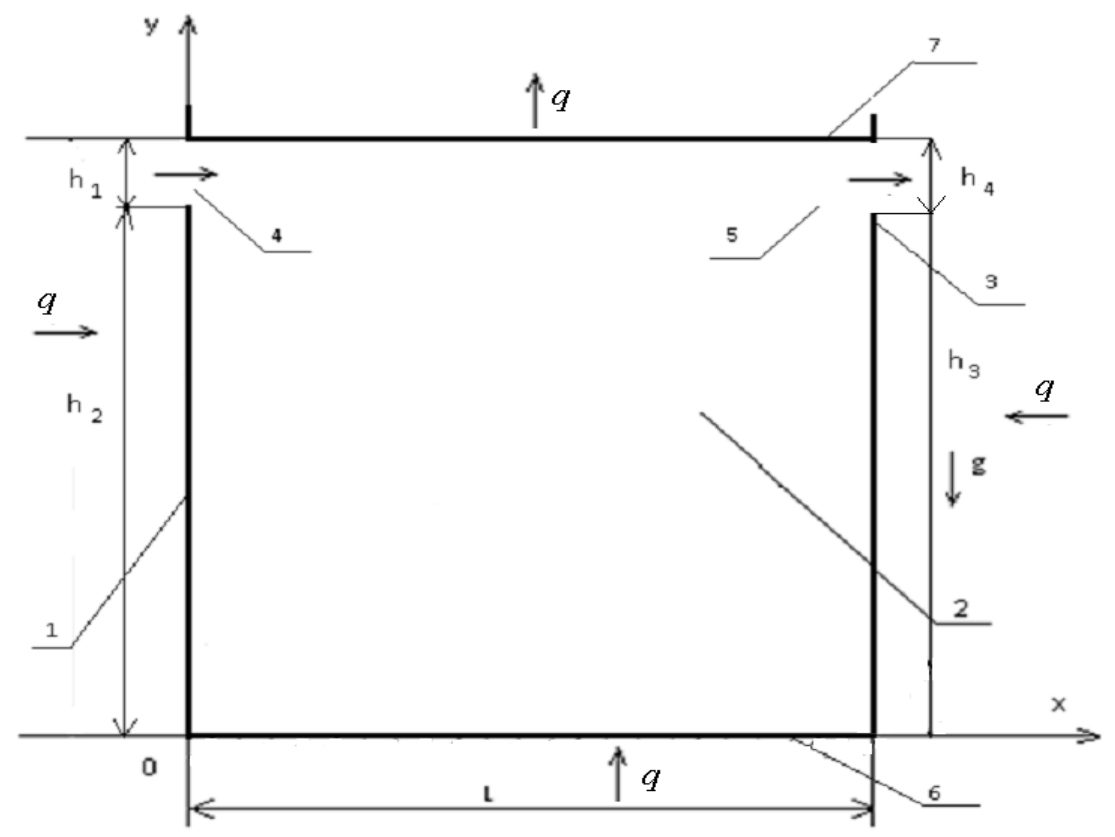

Figure 1. A scheme of the system

At the boundaries exhibited appropriate boundary conditions. It is assumed that initially the walls of the cavity and fills it with the fluid are constant and the identical at all points of the temperature, the liquid is stationary. It is assumed that the temperature of fluid injected is considered known and does not change with time. Mass inflow rate is also known value without changing the channel cross section and in time.

We consider several variants of the input and output sections of fluid mass in which substantially changes the structure of the flow in the storage tanks. Investigations were carried out at the following values of the dimensionless and dimensional quantities: $10^{4} \leq \mathrm{Gr} \leq 10^{6}, \mathrm{Re}=500, T_{0}=-162.4 \mathrm{~K}, T_{i n}=$ $-162.6 \mathrm{~K}, T_{e}=-161.5 \mathrm{~K}$. Ranges of the dimensionless quantities and dimensional characteristics were chosen based on the following conditions. The first-line range of parameters in real-world implementations of such systems. The second - the real extent of the existence of mixed convection. In addition, the controlled conditions of geometric similarity of real systems and used in the mathematical description of the process model. Distributions of hydrodynamic parameters and temperatures that characterize the basic laws of the process (Fig. 2-4).

The system of differential equations with appropriate initial and boundary conditions were solved by finite difference method. In solving this problem algorithm was used [7-10] developed for the numerical solution of natural convection in a closed rectangular areas with local sources of energy. 


\section{The results of numerical modeling}

Fig. 2-4 show typical results of solving the above problem for the mixed convection regime.

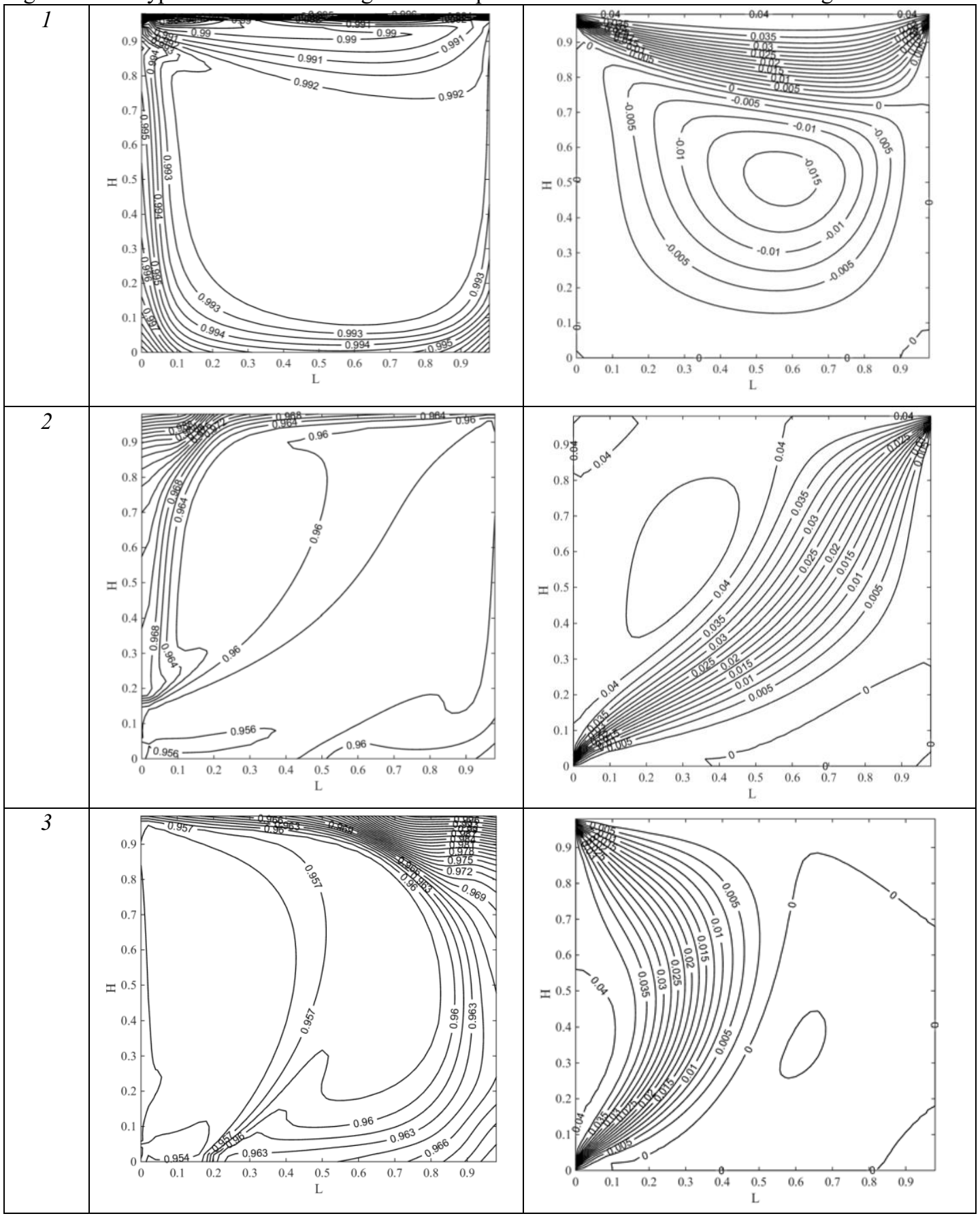

Figure 2. The temperature field and the contours of the stream function at different positions of the input and output sections of the fluid 


\section{MATEC Web of Conferences}

In the case of the location of the input and output sections of the masses in the upper portion of the cavity, the formation of a vortex in the central part of the tank as a result of the influence of both the forced and the natural motion of the medium. Figure 2 (2 and 3) is a division of the main flow cavity into two regions. Formed one vast vortex that pulls down the input stream. The main mechanism of the formation of this vortex is forced convection.

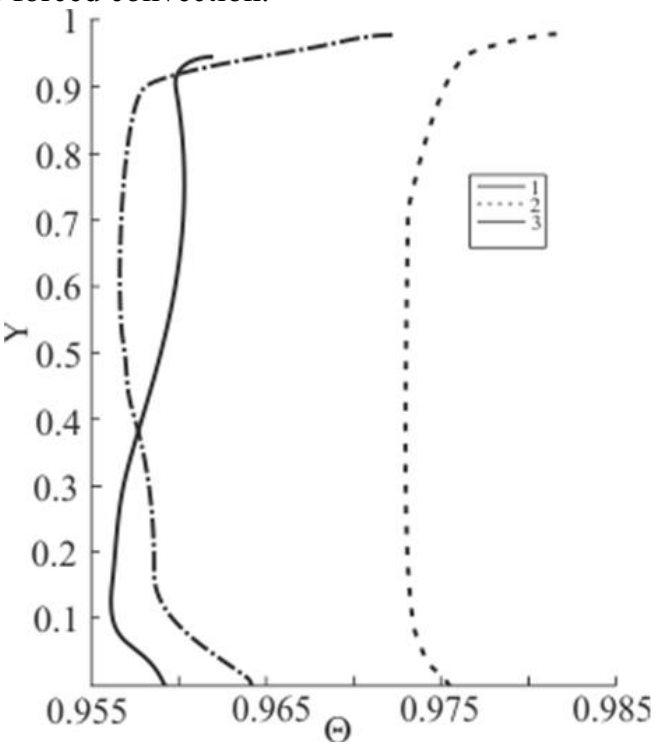

Figure 3. Temperature profile in the central section at a different location in the input and output portions of the liquid.
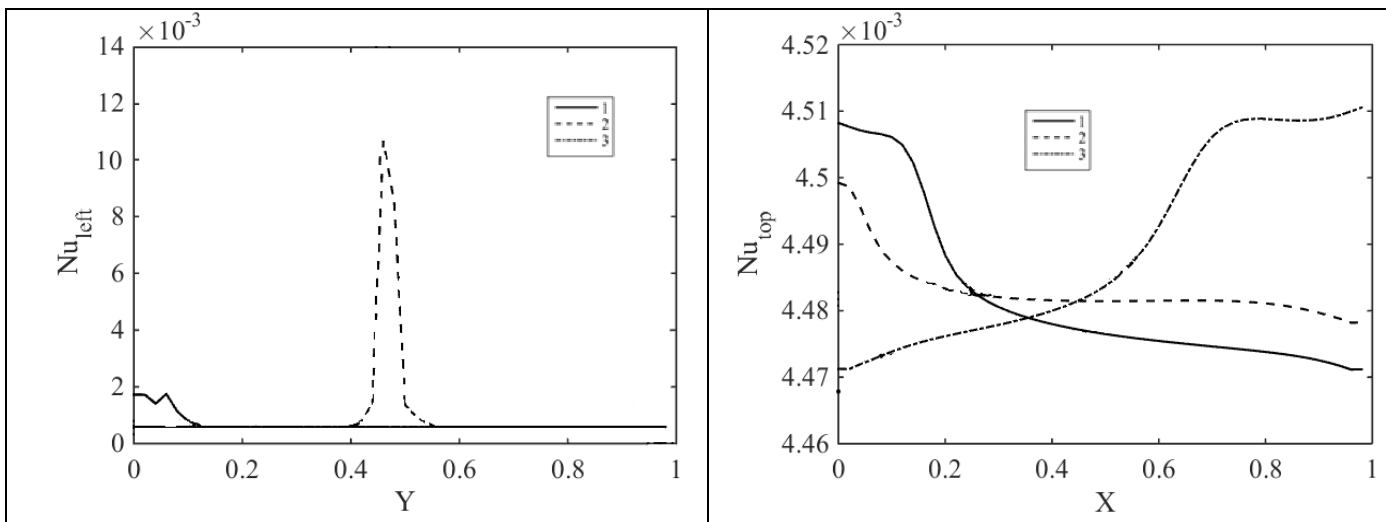

Figure 4. Distribution of the Nusselt number at different borders of the sites under consideration for the input and output mass

Figure 3 shows the temperature profile in the central section of the options under consideration for solving the problem. It is seen that the displacement input portion in a lower mass portion and the upper portion of the output is most intensive mixing of fluid in the reservoir due to the high intensity vortices is more uniform temperature distribution over the entire region of solutions, temperature drop height decreases.

The distribution of values of the Nusselt number on different boundaries of the solution region (Fig. 4) characterizes the heat transfer rate on the borders of the LNG storage tank. The upper 
boundary of solution heating is more intense than in other boundaries due to heat dissipation due to evaporation.

It is also worth noting that the Nusselt number on the free surface of the reservoir (Nu) (Fig. 4) is significantly higher. On the solid does not exceed the boundaries of $\mathrm{Nu} 6 \cdot 10^{-4}$, on the free as the number of Nusselt reaches $4,5 \cdot 10^{-3}$.

It was found that a change in the geometric arrangement of the input and output sections of the fluid changes the flow pattern in the cavity and the displacement inlet in the lower part of the temperature difference in height decreases.

\section{Conclusion}

Is theoretically investigated the influence of different source locations of input and output of mass into the low-temperature storage tanks LNG on the regimes of convective heat exchange. The results of work show the possibility of using the Navier-Stokes equations in the variables «vorticity - stream function» for the simulation of fairly complicated by nature flows with the moderate values of the numbers of Reynolds and Grashof in the regime of the mixed convection and with the heterogeneous heat exchange on the outer boundaries of the region of the solution.

On the basis obtained results it is possible to make a conclusion about the fact that, changing the coordinates of the sections of input and output of liquid it is possible to change the structure of flow and the thermal condition of low-temperature storage tanks LNG, and to also reveal stagnation zones and to estimate the effectiveness of the regenerative system of low-temperature storage of liquefied gases.

It is revealed, that a change in the speed of input flow also exerts a substantial influence on the structure of flow and temperature of liquid in the region being investigated. It is established that to regulate thermal condition in the low-temperature storage tanks LNG is possible also by a change in the circulating rate of liquefied natural gas.

The work was realized within the research state assignment "Science” №13.1339.2014/K (Code of Federal Target Scientific and Technical Program 2.1410.2014).

\section{References}

1. Bin Xu, Yumei Shi, Dongsheng Chen, Cryogenics, 60, 68 (2014)

2. M.W. Lukaszewski, W.B.J. Zimmerman, M.T. Tennant, Chem. Eng. Res. Des., 91, 3 (2013)

3. Calogero Migliore, Cristina Tubilleja, Velisa Vesovic, J. Nat. Gas Sci. Eng., 26, 570 (2015)

4. O.N. Aneziris, I.A. Papazoglou, M. Konstantinidou, Z. Nivolianitou, J. Loss. Prevent. Proc., 28, $23(2014)$

5. Yuxing Li, Zhenglong Li, Wuchang Wang, J. Loss. Prevent. Proc., 37, 132 (2015)

6. Lu, X.S., Lin, W.S., Gu, A.Z., Qin, Z.H., Numerical modeling of stratification and rollover in LNG and the improvements to Bates-Morrison Model (In: Proceedings of the 6th ASME-JSME Thermal Engineering Joint Conference, Kohala Coast, Hawaii, 2003)

7. G.V. Kuznetsov, V.I. Maksimov, Journal of Engineering Thermophysics, 4 (16), 1883 (2007)

8. G.V. Kuznetsov, N.I. Kurilenko, V.I. Maksimov, G.Ya. Mamontov, T.A. Nagornova, Journal of Engineering Physics and Thermophysics, 86, 3 (2013)

9. G.V. Kuznetsov, M.A. Sheremet, Thermophysics and Aeromechanics, 16, 1 (2009)

10. V. I. Maksimov, T.A. Nagornova, EPJ Web of Conferences, 76, (2014) 